\title{
Determining Design Parameters for Ad Hoc Wireless Sensor Networks
}

\author{
Qiang Gao, Keith J. Blow, David J. Holding \\ Aston University \\ Aston Triangle \\ Birmingham, B4 7ET \\ U.K.
}

\begin{abstract}
This paper considers the design of wireless sensor networks in which a set of smart battery-powered sensor nodes cooperatively form an ad hoc communications network for monitoring and control applications. The paper examines ways of extending the life of such networks by introducing a 'sleep mode' in the sensor node. Quantitative analysis is used to show that although there is a complex relationship between 'sleeping' and energy conservation, it is possible to make significant energy savings while incurring only modest degradation in performance. An optimum energy saving curve is derived which provides a basis for the design of extended-life ad hoc wireless sensor networks.
\end{abstract}

\section{INTRODUCTION}

The idea of using a loosely-coupled set of self-organising intelligent sensors is attractive in a range of monitoring, control, and surveillance systems. The concept becomes particularly appealing when wireless communications technologies are used to interconnect the sensors. Arguably the most flexible wireless sensor network is formed when a distributed set of smart sensor nodes are networked together in an ad-hoc fashion. The smart sensor nodes are autonomous devices equipped with heavily integrated sensing, processing, and wireless communication capabilities [1][2]. The set of sensor nodes are essentially self-organizing wireless hosts that cooperatively form an ad hoc communications network without the need for a fixed infrastructure. The nodes of such a wireless sensor network gather data via their sensors, process it locally or coordinate amongst neighbours and forward the information to a user or controller. In many applications, such as monitoring, the wireless sensor network is event driven in the sense that it is designed to monitor sensor inputs, detect of interest, and report such events with associated data.

Recent advances in micro-electro-mechanical systems (MEMS) technology, wireless communications and digital electronics have enabled the development of low-cost, lowpower, multi-functional smart sensor nodes that are generally powered by non replaceable energy sources such as small batteries [3]. In such systems, the wireless communication capability is normally the main consumer of energy and energy efficiency is a critical design constraint. Research can address two different perspectives of the energy problem: first to increase battery capacity while satisfying size and weight constraints, and second to decrease the amount of energy consumed at the wireless terminal. The first goal primarily depends on advances in battery technology. More flexible and innovative results may be obtained in a shorter timescale by focussing on the second goal of decrease the energy consumed in the ad hoc wireless network [4].

\author{
Ian Marshall \\ BTexacT \\ Adastral Park, Martlesham Heath \\ Ipswich, Suffolk, IP5 3RE \\ U.K.
}

This paper addresses the problem of extending the operational life of ad hoc sensor networks by conserving battery energy. In particular, the paper examines the notion of minimizing the energy used by the wireless part of the event-driven sensor nodes by letting the wireless 'sleep' when the node is simply monitoring environmental parameters. It follows that such a node can be either sleeping, where no transmission or reception can occur, or awake where traffic is processed. However, increasing the sleeping time does not always lead to an increase in the energy saved. There is a complex relationship between sleeping time and energy consumption. The research reported in this paper uses quantitatively analyses the trade offs between energy conservation and quality of service in an ad-hoc wireless sensor network. The results of the research identify the energy saving profile for various levels of sensor network activity and the limits for such an energy conservation approach.

\section{DESCRIPTION OF A SENSOR NODE}

A primary task of a node in an ad hoc sensor network is to sense its environment and processing the information for onward transmission. Due to the limited transmission range of low-cost low-powered nodes, this forwarding mostly involves using multi-hop paths through other nodes [1]. Therefore, a general sensor node also has the task of forwarding traffic from other sensors as an intermediate relay in the multi-hop path. In terms of energy consumption, the wireless exchange of data between nodes strongly dominates other node functions such as sensing and processing [5][6]. Research in location-aware and context-aware ad hoc networks has addressed the problem of usefully reducing the transmit-power of a node's radio to match the hop-distance to the adjacent node and the transmission environment [7][8]. However, radios consume power not only when sending and receiving data, but also when listening and energy models have been developed which show that the energy consumption ratio of listen:receive:send is about $1: 1: 1.5$ [7][8]. In a typical monitoring application, traffic in the event driven sensor network is normally light and node listening time dominates energy consumption. Therefore, significant energy savings are only obtainable by putting the node wireless into a sleep mode when there is no traffic [9]. 


\section{A. Developing a model of a sensor node}

Consider a network of wireless sensor nodes where each node consists of a transmitter and receiver together with some sensing device. Let the sensor node be kept as simple as possible in order to enable mass deployment and assume that

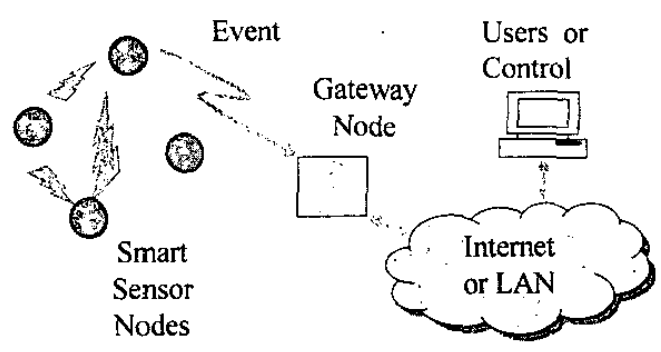

Fig. 1 Fragment of a sensor network

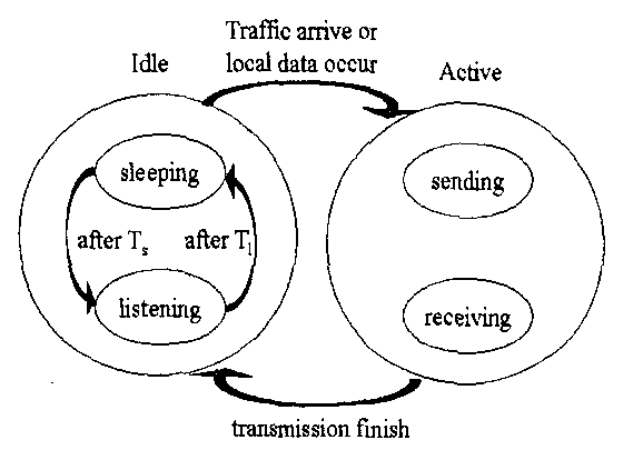

Fig. 2 State diagram of the sensor node radio system

a single antenna is available (thus the node can transmit or receive but not both simultaneously). The sensing device is a source of information flow local to the sensor node and each sensor node will accept information from other nodes for onward transmission. There will also be nodes in the network, such as user or control nodes, where information terminates (i.e. information 'sink' nodes) as shown in Fig. 1. These will not be explicitly modelled as the focus of interest is in the relationships between local and transit traffic and how these affect the performance of the network. Generally the nodes will have limited computational and memory resources; therefore the queuing of packets in the node will be modelled explicitly.
To achieve a low energy target, let the radios sleep most of the time and awaken when they need to transmit or receive data. Since current radio technology does not easily allow a radio to be awakened upon request, a radio must wake up periodically to see if other nodes want to send to it; if it is not required then it may go back to sleep. The four possible states of the sensor nodes are shown in Fig. 2. A node is classified as 'active' when it is in a transmitting or receiving state and as 'idle' when it is in either a listening state (i.e. listening but not actually receiving data) or a sleeping state (i.e. not listening to the outside world although the sensors and other low-power parts of the node may be on). These four states have different energy consumptions: let the energy consumption ratios to be listen : receive: send $=1: 1: \mathrm{b}$ (this assumes that the radio energy consumption is zero in the sleeping state).

Transitions between these states can occur naturally, such as the change from listening to receiving when data arrives from a neighbouring node, or as a result of some internal decision, such as the scheduling algorithm used to decide when to change from listening to sleeping and vice versa. There are additional subtleties associated with sources of energy consumption, such as discriminating in the transmit state between the energy consumption associated with establishing a connection and the energy consumption associated with the process of actually sending packets. These subtle distinctions often involve significant amounts of energy, lead to complex relationships between energy saved and sleeping time, and increase the complexity of the energy minimization problem.

\section{TOWARDS AN ENERGY CONSERVING ALGORITHM}

Let the nodes be initialised in the 'idle' sleeping state. When a node is asleep, if sensor data is generated that has to be transmitted then the node changes to the 'active' transmit state and starts sending the data. However, if a node remains asleep for time $T_{s}$ then it undergoes a transition to the 'idle' listening state. During the listening state if neighbouring nodes try to transfer packets via the node, or if sensor data is generated that needs to be sent, then the node changes to the appropriate 'active' state. Otherwise it returns to the sleeping state after time $\mathbf{T}_{1}$. When a node is in an 'active' state it is either sending or receiving data. After all data transmission has finished, the node immediately changes to an 'idle' state and begins alternately sleeping and listening according to the predefined schedule. Thus, the total radio energy consumption is the sum of (i) the energy consumption of periodically listening when the node is idle, (ii) the energy consumption when receiving data, and (iii) the energy consumption when the node is in the sending state (comprising the energy consumption for establishing a connection before the sending operation and the energy consumption for the actual process of sending packets). 
The aim of the algorithm that turns off the radio is to conserve this energy. However, turning off the radio has implications for the performance of the sensor network such as added latency, reduced throughput, and possibly increased packet loss compared to the communication protocols without sleeping patterns. Therefore, in studying potential energy savings it is necessary to characterize the trade-off in performance and identify optimal control of the sleep/listen pattern.

The research assumes that the individual nodes are asynchronous and that sensor traffic occurs randomly. These assumptions facilitate the performance analysis of a simple asynchronous algorithm and provide a base level of performance for judging the effectiveness of synchronous or other more complex algorithms. For example, it provides a metric for evaluating the energy efficiency and performance of synchronisation schemes such as local entrainment with local synchronised clusters or time-based synchronisation.

\section{ANALYSIS OF THE SENSOR NETWORK}

The analysis of the problem comprises three stages. First, telecommunications theory is used to calculate the effect of the algorithm that turns off the radio on the performance (connection probability, throughput, packet loss and delay) of the ad hoc network. Second, the effect of the algorithm on energy consumption in the network is determined. Finally, an optimum energy strategy is developed for the ad hoc sensor network.

Consider the case when Node 1 needs to transfer data (local sensor data or data needing to be forwarded from neighbouring nodes) to Node 2. Let this traffic be $A$ Erlangs. At Node 1 let the number of packets waiting in the queue be $x$ and the number of packets in onward transmission be $s$; let $[x, s]$ denote the state of the sending node; let $u(x, s)$ denote the probability that a packet begins to be transmitted after a packet arrives at state $[x, s]$; and let $v(x, s)$ denote the probability that, after a packet finishes transmission, another packet begins to be transmitted. They are expressed as the following respectively

$$
u(x, s)=\left\{\begin{array}{l}
T,(s=0) \\
0,(s=1)
\end{array} \quad v(x, s)=\left\{\begin{array}{c}
T,(x \geq 1, s=1) \\
0,(x=0 \text { or } s=0)
\end{array}\right.\right.
$$

where $s \in\{0,1\} ; 0 \leq x \leq K$ and $K$ is the length of waiting queue, and $\mathrm{T}$ is the connection probability from Node_1 to Node 2.

The connection probability $\mathrm{T}$ is determined by the sleeping time and listening time of the receiving node, Node 2, and the traffic between Node 1 and Node 2 . Each sensor node performs a dual function of sending out local sensor data (this involves use of the transmitter only) and relaying data sent from other nodes (involve the use of both the receiver and the transmitter). Since our simple nodes have a single antenna they cannot simultaneously transmit and receive simultaneously and the model must account for the mutual blocking of these two processes.
The research considered two priority schemes, (i) the sending operation has higher priority than the receiving operation, and (ii) the receiving operation has higher priority than the sending operation. Calculations show that under heavy traffic conditions, when there will generally be some packets in the waiting queue of the receiving node, the first scheme results in very low throughput. Consideration therefore turned to the second prioritisation in which the sending node can successfully connect to the receiving node when there is no packet in the waiting queue of the receiving node and it is in listening state or when there are some packets in the waiting queue but the receiving node is not actually in the process of sending packets. In this case the formula for the connection probability becomes:

$T=T_{0} p(0,0)+\left(1-p(0,0)-A^{\prime}\right)$

Where $A^{\prime}$, note $0<A^{\prime}<1$, is the carried traffic and $T_{0}=\frac{T_{1}}{T_{s}+T_{1}}$ represents the sleeping time factor of the receiving node, and $T_{l}, T_{s}$ are the listening time and sleeping time respectively. The first term on the right is the contribution of listening state and the second term is the contribution when there are some packets in the queue but the receiving node is not actually sending information.

Assume that the traffic can be described by Poisson distributed arrival times and negative exponential holding times (the holding time corresponds to the packet length), then we can obtain general equations of statistical equilibrium [10]. Let $p(x, s)$ be the probability of the state $[x, s]$, then for a very short interval time $d t$ the probability of a packet arrival is Adt (terms in higher powers of $d t$ are neglected) and the probability that a packet finishes transmission is sdt. The statistical equilibrium assumption that $p(x, s)$ is independent of the time implies that the state $p(x, s)$ is created as often as it is destroyed and the general equations of statistical equilibrium are as follows:

$$
\begin{aligned}
& (A+s) p(x, s)= \\
& A[1-u(x-1, s)] p(x-1, s) \\
& +A u(x, s-1) p(x, s-1) \\
& +(s+1)[1-v(x, s+1)] p(x, s+1) \\
& +s v(x+1, s) p(x+1, s)
\end{aligned}
$$

The left-hand side of the equation represents the rate at which the system leaves the state $[x, s]$ due to packet arrival rate $A p(x, s)$ and packet finishing transit rate $s p(x, s)$. The first term on the right represents the rate at which the system leaves the state $[x-1, s]$ and enters $[x, s]$; the second term represents the rate at which it leaves $[x, s-1]$ and enters $[x, s]$; the third and the forth terms represent the rate at which it leaves $[x, s+1]$ or $[x+1, s]$ and enters $[x, s]$ respectively.

To give the solution we have the further standard assumption that: transition probabilities from state $[x-1, s]$ to 
state $[x, s]$ and from $[x, s-1]$ to $[x-1, s]$ are the same as those from $[x, s]$ to $[x-1, s]$ and from $[x, s]$ to $[x, s-1]$, respectively. The following expressions are then obtained for the probability of the state $[x, s]$ that $x$ packets are in the queue and $s$ packets are in transit.

$$
\begin{aligned}
& p(x, 0)=\frac{A^{x}(1-T)^{x}}{T^{x}} p(0,0) \\
& p(x, 1)=A^{x+1} T p(0,0) \\
& +\sum_{i=1}^{x} \frac{A^{x+1}(1-T)^{i}}{T^{i-1}} p(0,0)
\end{aligned}
$$

The probability of packet loss is:

$$
L=p(K, 1)+p(K, 0)
$$

the carried traffic is:

$$
A^{\prime}=A[1-p(K, 1)-p(K, 0)]
$$

and the average delay time (i.e. the waiting time averaged over the carried traffic), is:

$D=\frac{\bar{q}}{A^{\prime}}$

where the averaged number of waiting packets is:

$\bar{q}=\sum_{x=1}^{K} x p(x, 1)+\sum_{x=1}^{K} x p(x, 0)$

The total energy consumption of one node can then be expressed as:

$E=A^{\prime}+\left(1-A^{\prime}\right) p(0,0) T_{0}+b\left(1-A^{\prime}\right)[1-p(0,0)]$

Where the first term on the right, $A^{\prime}$ is the energy consumption for data receiving; the second term $\left(1-A^{\prime}\right) p(0,0) T_{0}$ is the energy consumption for listening, and the third term $b\left(1-A^{\prime}\right)[1-p(0,0)]$ is the energy consumption when the node is in the sending state. There are two parts in this last term, $b A^{\prime}$ the energy consumption for the actual process of sending packets, and $b\left(1-A^{\prime}\right)[1-p(0,0)]-b A^{\prime}$, the energy consumption for establishing a connection before the sending operation.

\section{ENERGY CONSERVATION VERSUS QUALITY OF SERVICE}

Simulation results show that, as expected, conserving energy leads to a reduction in network quality of service. Specifically, when the energy conservation algorithm is applied to an ad hoc sensor network with waiting queue length $K=5$ the packet loss rate $\mathrm{L}$ becomes higher for higher values of the sleeping time $T_{s}$ (i.e. smaller $T_{0}$ ), Fig. 3.

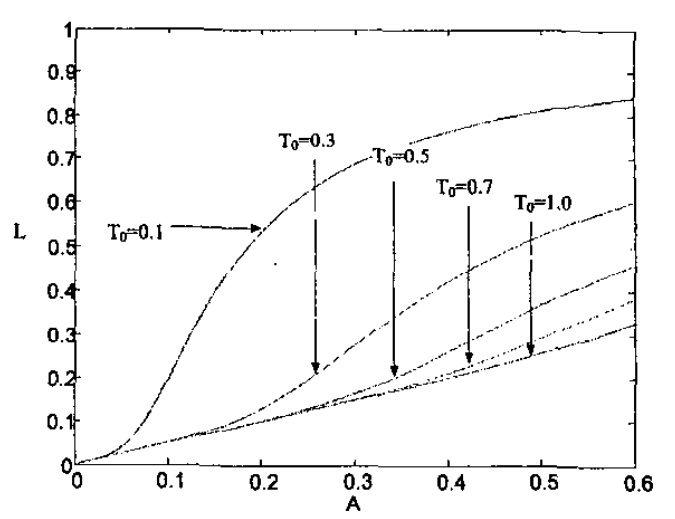

Fig. 3 Packet loss ratio against traffic

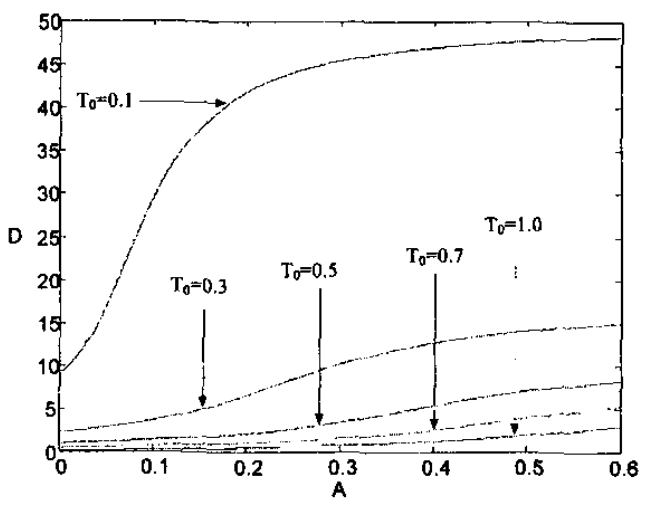

Fig. 4 Packet delivery delay against traffic

Significantly, for ad hoc sensor networks with real-time monitoring and control functions, the packet delay increases with increased sleeping time, as shown in Fig. 4. In all the above cases, values of $T_{0}$ in the range $0.3-0.5$ give much better results than $T_{0}=0.1$.

Fig. 5 shows the total and functional energy consumption for a sensor node (receiving queue length $\mathrm{K}=5$, relative transmit energy $\mathrm{b}=1.5$ ) when listening, receiving data, sending data, and establishing a connection, when $T=0.3$ and $T=0.5$. It can be seen that for both values of $\mathrm{T}$ the energy consumption for establishing a connection is much bigger than the energy consumption for other states such as listening, sending and receiving data. This is the main reason for the complex relationship between energy saved and sleeping time, and explains why a longer sleeping 


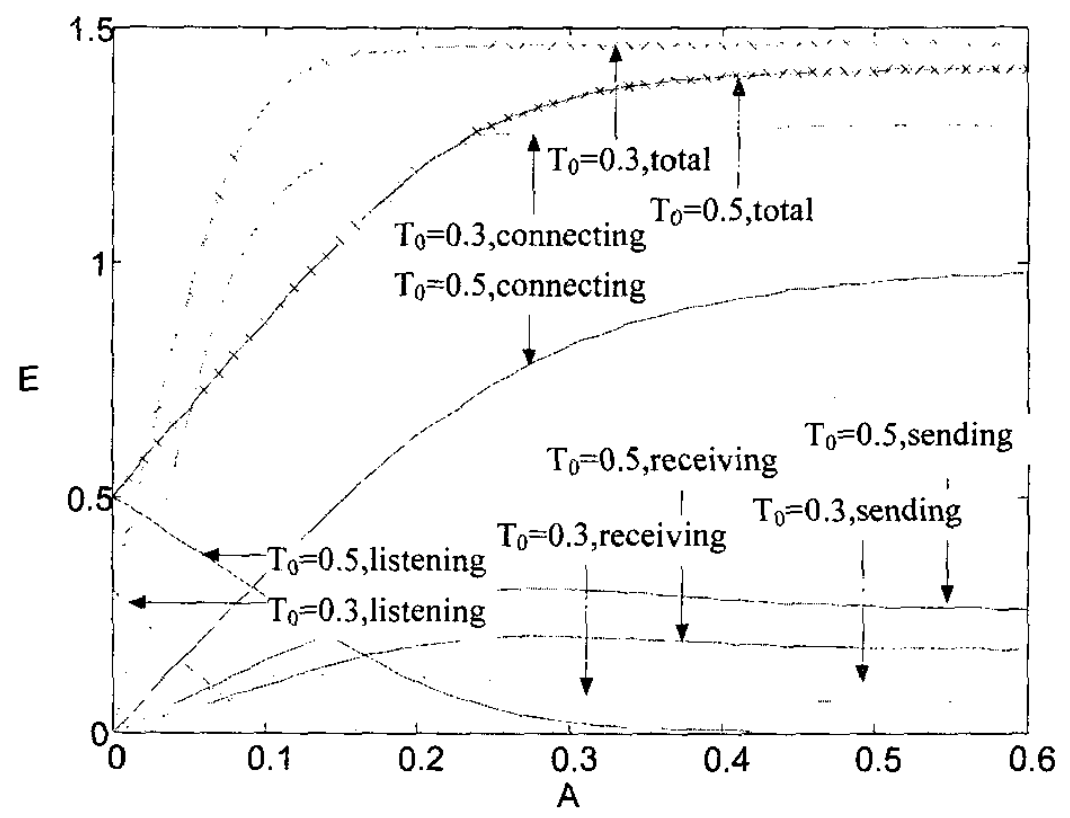

Fig. 5 Causal analysis of energy consumption

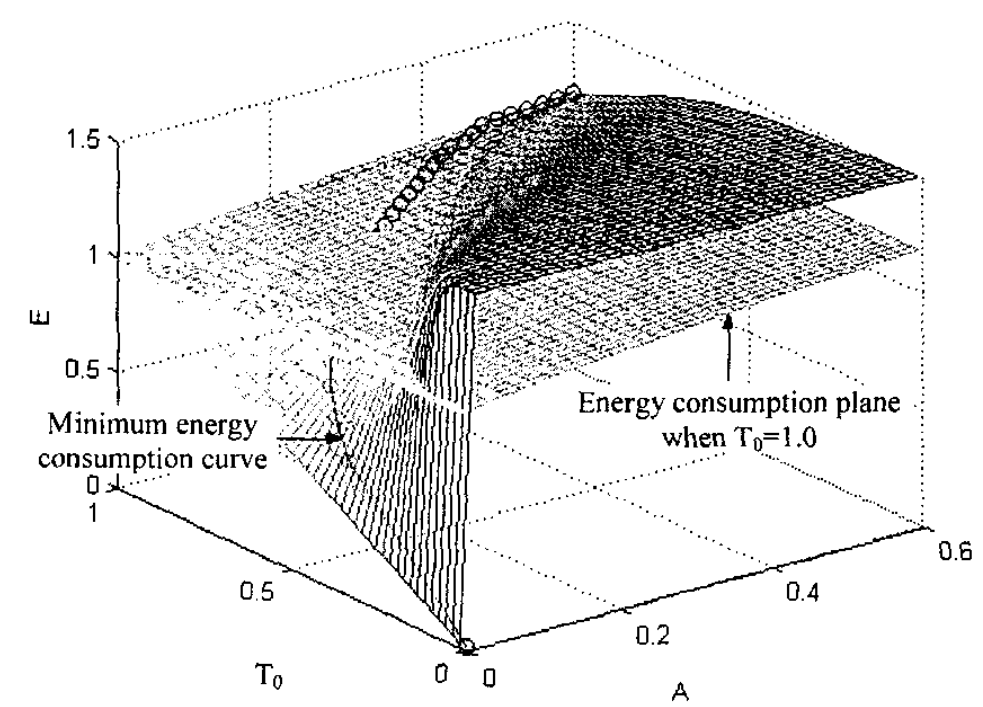

Fig. 6 Minimum energy consumption curve 
time does not always lead to an increase in energy saving. Fig. 6 shows the surface of energy consumption $E$ $(K=5, b=1.5$ ) versus the amount of traffic $A$ and the sleeping time factor variable $T_{v}$.

Using the energy conserving (sleeping) algorithm, actual energy savings can be made only in the part of the energy consumption surface under the energy consumption plane, $\mathrm{T}=1.0$, which corresponds to a nosleeping schedule in the radio. The optimum energy saving curve (i.e. minimum energy consumption curve) is shown clearly in Fig. 6 . This shows that in ad hoc sensor networks, a sleeping schedule can be chosen that provides significant energy saving while potentially delivering acceptable levels of quality of service including communication packet delay.

\section{CONCLUSIONS}

In wireless ad hoc sensor networks, where energy efficiency is the key design challenge, the energy consumption is typically dominated by the node's communication subsystem. Only switching the sensor node radio into a sleep state (if there is no sensor data to report or no multi-hop network traffic to transfer) can reduce the energy consumption significantly. However introducing sleeping time will reduce throughput and increase packet loss and delivery delay,

Quantitative analysis of the complex relationship between energy consumption, quality of service and sleeping time shows that with careful selection of the sleeping schedule it is possible to make significant energy savings while incurring only modest degradation in the quality of service. An optimum energy saving curve (i.e. minimum energy consumption curve) has been derived and this provides a basis for the design of extended-life ad hoc wireless sensor networks.

\section{REFERENCES}

[1] L. Clare, G. Pottie and J. Agre, "Self-organizing distributed sensor networks", in Proc. SPIE Conf. on Unattended Ground Sensor Technologies and Applications, vol. 3713, 2000, pp. 229-237.

[2] K. Sohrabi, J. Gao, V. Ailawadhi and G. Pottie, "Protocols for self-organization of a wireless sensor network," IEEE Personal Communications Magazine, vol. 7, no.5, 2000, pp. 16-27.

[3] I.F. Akyildiz, W. Su, Y. Sankarasubramaniam and E. Cayirci, "Wireless Sensor Networks: A Survey", Computer Networks, vol. 38 , no. 4, 2002, pp. 393422.

[4] P. Lettieri and M. Srivastava, "Advances in wireless terminals", IEEE Personal Communications; vol. 6, no. 1, 1999, pp. 6-18.

[5] D. Estrin and R. Govindan, "Next century challenges: scalable coordination in sensor networks", in Proc. MobiCom 1999, pp. 263-270.

[6] A. Savvides, C. C. Han and M. Srivastava, "Dynamic fine-grained localization in ad-hoc networks of sensors", in Proc. MobiCom 2001, pp. 166-179.

[7] S. Narayanaswamy, V. Kawadia, R. S. Sreenivas, and P. R. Kumar, "Power Control in Ad-Hoc Networks: Theory, Architecture, Algorithm and Implementation of the COMPOW Protocol", in Proc. European Wireless Conference 2002, pp.156162.

[8] A. Schmidt, K. A. Aidoo, A. Takaluoma, U. Tuomela, K. V. Laerhoven and W. Van de Velde, LNCS 1707, Springer, Germany 1999, pp 89.

[9] C. E. Jones, K. M. Sivalingam, P. Agrawal and J. C. Chen, "A survey of energy efficient network protocols for wireless networks", Wireless Networks, vol. 7, no. 4,2001, pp. 343-358.

[10] D. Bear, Principles of Telecommunication Traffic Engineering, Peter Peregrinus Ltd. U.K;1988.

[11] M. Stemm, and R. H. Katz, "Measuring and reducing energy consumption of network interfaces in hand-held devices", IEICE Transactions on Communications, vol. E80-B, no.8,1997, pp. 1125 1131. 